\title{
Potentiometric Biosensing Applications of Graphene Electrodes with Stabilized Polymer Lipid Membranes
}

\author{
Georgia-Paraskevi Nikoleli ${ }^{1, *}$, Dimitrios P. Nikolelis ${ }^{2}{ }^{(0)}$, Christina G. Siontorou ${ }^{3}$, \\ Marianna-Thalia Nikolelis ${ }^{3}$ and Stephanos Karapetis ${ }^{1}$ (i) \\ 1 Laboratory of Inorganic \& Analytical Chemistry, School of Chemical Engineering, Dept 1, \\ Chemical Sciences, National Technical University of Athens, 9 Iroon Polytechniou St., \\ 15780 Athens, Greece; stevekara@chem.uoa.gr \\ 2 Laboratory of Environmental Chemistry, Department of Chemistry, University of Athens, \\ Panepistimiopolis-Kouponia, 15771 Athens, Greece; nikolelis@chem.uoa.gr \\ 3 Laboratory of Simulation of Industrial Processes, Department of Industrial Management and Technology, \\ School of Maritime and Industry, University of Piraeus, 18534 Piraeus, Greece; csiontor@unipi.gr (C.G.S.); \\ m.nikolelis@gmail.com (M.-T.N.) \\ * Correspondence: dnikolel@chem.uoa.gr
}

Received: 25 May 2018; Accepted: 27 June 2018; Published: 28 June 2018

\begin{abstract}
This review provides information and details about the fabrication of biosensors composed of lipid membranes that can be used to rapidly detect toxic compounds in food, environmental pollutants, and analytes of clinical interest. Biosensors based on polymeric lipid membranes have been used to rapidly detect a wide range of these analytes, offering several advantages including fast response times, high sensitivity and selectivity, portability for field applications, and small size. A description of the construction of these devices and their applications for the rapid detection of toxic substances in food, environmental pollutants, and analytes of clinical interest is provided in this review.
\end{abstract}

Keywords: biosensors; lipid membranes; potentiometry; graphene electrodes

\section{Introduction}

A biosensor is an instrument that analyses a sample and provides its chemical concentration and composition. A chemical sensor consists of two parts: a biological element that chemically recognizes the unknown compound and an element that provides the chemical information in a physical unit that transforms the signal into a measurable electrical, optical, or piezoelectric signal (e.g., voltage, current, absorbance, etc.). The advantages of biosensors compared to classical analytical devices, such as liquid and gas chromatography, are as follows: faster response times, higher throughput of samples, smaller size, and portability for field measurements. It is also cheaper and personnel do not require training for its use.

Chemosensors are similar to biosensors but are composed of a synthetically prepared molecule instead of a biological element. They recognize small molecules or metal ions by binding to them. The synthesis, design, and applications of chemosensors are of special interest for the detection of analytes. The present review provides an outline of applications of chemosensors that have not been well covered elsewhere, such as the design and synthesis of calixarenes that have been used to rapidly determine carbamates [1] and naphthalene acetic acid (NAA) [2].

Nanobiosensors are based on the merging of nanotechnology with biosensors. Such materials include graphene, carbon nanotubes, and nanowires. Metal-based nanoparticles are also excellent hosts for the construction of electrical and optical devices that can be applied to detect nucleic acid 
sequences. Various nanomaterials have been explored and their properties were analyzed for possible applications in biosensors. Nanobiosensor technology research has prompted the construction of an increasing number of novel devices.

This paper addresses the fabrication of biosensing devices composed of lipid membranes on graphene electrodes that have been used and applied to rapidly detect toxic substances in food, environmental pollutants, and analytes of clinical interest. These devices are fabricated from a polymerized lipid films on graphene electrodes. The construction of these devices and their applications in the rapid detection of the above analytes are described in this paper [3]. Figure 1 provides a schematic of a lipid-based biosensor on a graphene electrode that has been used for the potentiometric detection of urea

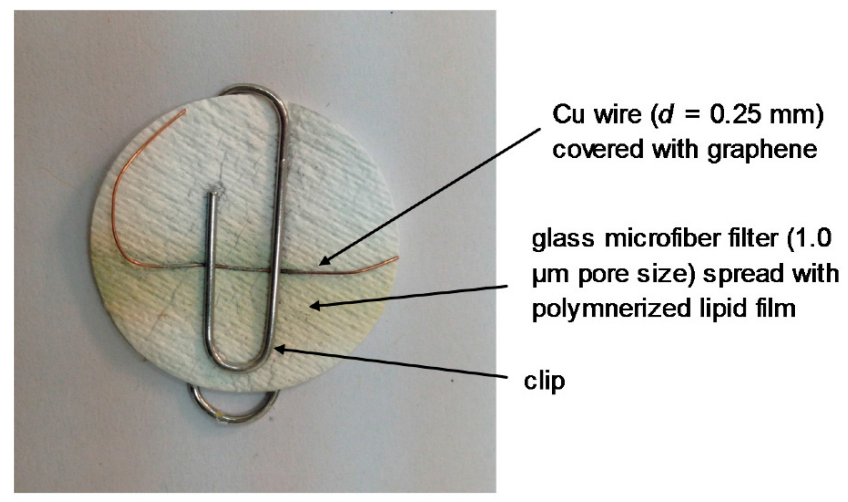

Figure 1. Schematic of a lipid membrane-based biosensor on a graphene electrode. This device was used for the potentiometric determination of urea. Reprinted with permission from Nikoleli et al. [3].

\section{Polymer Lipid Membranes}

Stabilized in electrolyte solution, lipid film-based biosensors were first constructed by Nikoleli et al. [4] by using glass fiber filters to support and stabilize the lipid membrane. However, these membranes were not stable outside an electrolyte solution in the air. Later on, lipid polymer membranes supported on glass fiber were shown to be stable in air [5]. The deposition of these polymer lipid films on graphene electrode [3] offered many advantages such as increased sensitivity and selectivity, rapid response times, and provided remote sensing biosensors for urea.

Biosensors have been developed since the 1970s and a pioneer in this field of science was Turner. Lipid membrane biosensors appeared in 1990 with the work of Krull and Nikolelis [4]; Hianik introduced lipid membrane-based biosensors focused mainly on metal supported lipid films. Nanotechnology has offered a method for preparing nanosensors based on lipid membranes by using graphene electrodes. Biosensors that are based on lipid membranes provide many advantages over the standard analytical methods (i.e., chromatography or mass spectroscopy), including rapid response times, small size, low cost, time efficiency, portability for use in the field, and most importantly, biosensors are biocompatible. Advances in the construction of air-stable lipid films have allowed their application to the detection of a wide range of compounds that are toxic in foods or are of environmental or clinical interest.

The design, construction, and storage of polymer lipid membranes on microporous filtering media such as glass fibers has been described extensively in one of our previous papers [5]. We examined the simplification of membrane construction and the stability of these lipid membranes after they were stored in air so that they could be repetitively used many times. The preparation technique for the polymer lipid membranes has been standardized as follows: $5 \mathrm{mg}$ of phosphatidyl choline (PC) is stirred in a small vial with $0.070 \mathrm{~mL}$ methacrylic acid, $0.8 \mathrm{~mL}$ ethylene glycol dimethacrylate, $8 \mathrm{mg}$ 2,2'-azobis-(2-methylpropionitrile), and $1.0 \mathrm{~mL}$ acetonitrile. The roles of methacrylic acid, ethylene glycol dimethacrylate, and 2,2'-azobis-(2-methylpropionitrile) (AIBN) were explained in one of our 
previous paper [5]. Nitrogen was passed through this mixture by sparging for about one minute, then was sonicated for half an hour, and stored in the refrigerator. To prepare stabilized polymer lipid membranes, $0.15 \mathrm{~mL}$ of this mixture was injected onto the surface of a microfiber filter and was then irradiated with an ultraviolet (UV) deuterium lamp for four hours. This polymer lipid film can be directly used to construct devices based on lipid membranes.

These stabilized polymeric lipid membrane-based biosensors can be used as host matrices to maintain the activity of a biological compound and for the activity transduction of a wide number of biological active compounds, such as enzymes, antibodies, and artificial or natural receptors [5]. These devices are stable in air for more than one month and respond to a wide range of compounds and analytes [6-8]. The advantages of polymerization by irradiation by using a UV deuterium lamp, instead of heating at $60^{\circ} \mathrm{C}$ for our hours, has been described in one of our previous papers [9].

In this paper [9], UV irradiation was used instead of heating the mixture at $60^{\circ} \mathrm{C}$ because this process retains the activity of the enzyme, antibody, or receptor. Heating may deactivate these biological receptors. The method used to prepare stable biosensors based on lipid membranes by heating at $60{ }^{\circ} \mathrm{C}$ was empirical; however, the polymerization mechanism was never investigated. We explored the preparation technique for stable lipid films by using physicochemical methods such as Raman and infrared (IR) spectrophotometry, differential scanning calorimetry (DSC), and scanning electron microscopy (SEM) experiments [9]. The results of Raman and IR showed that the polymerization kinetics ended after four hours. The polymerization mechanism and generation of the signal was further on explored by Raman and IR spectroscopy, micro-Raman spectroscopy, DSC, and SEM experiments $[2,3,9,10]$. These membranes were stable for storage in air for a period of longer than two months.

This process enabled the practical use of biosensors based on lipid membranes because the technique allowed the incorporation of natural ion-channels in these devices and will eventually permit these devices to be commercialized.

This UV light irradiation procedure was shown to retain the activity of acetylcholinesterase for the detection of carbamates [9]; heating at $60{ }^{\circ} \mathrm{C}$ would have caused deactivation. An important criterion for practical applications of lipid membrane-based biosensors is the ability to store these membranes log-term at a temperature of $25^{\circ} \mathrm{C}$. A number of practical characteristics of these lipid membrane-based biosensors, such as stability at ambient temperatures, reproducibility, and reusability, have demonstrated their analytical applications in our other reports [9-12].

\section{Polymer Lipid Membranes on Graphene Electrode}

We reported a method for constructing devices based on polymeric lipid films incorporated in a graphene electrode [3]. This technique is as follows: a homogeneous dispersion (ca. $0.4 \mathrm{mg} / \mathrm{mL}$ ) of graphene in a solvent $\mathrm{N}$-methyl-pyrrolidone (NMP) was sonicated for ca. $180 \mathrm{~h}$ and was then centrifugated at $700 \mathrm{rpm}$ for two hours [13]. The prolonged sonication time was requisite to reduce the size of the flakes, which is a requirement for many applications.

At this stage, this suspension was deposited on a copper wire with a diameter of $0.25 \mathrm{~mm}$, which was placed on a glass fiber filter and the organic solvent was evaporated by a fan heater. The wire was used to help the connection in order to provide the voltage as the output of the signal.

The receptor molecules were incorporated in the lipid films at the polymerization stage by spreading $10 \mathrm{~mL}$ of a receptor suspension onto the polymerization mixture. For instance, in order to prepare devices for the detection of cholera toxin, $10 \mathrm{~mL}$ of the ganglioside suspension, which acts as a receptor in this case, was mixed with $0.15 \mathrm{~mL}$ of the polymerization mixture using a microsyringe at the surface of the microfiber filter. The graphene electrodes with incorporated lipid membranes were stored at $4{ }^{\circ} \mathrm{C}$ when not in use for periods of more than three months.

The construction of the potentiometric biosensor concluded after the encapsulation of the filter-supported polymer lipid membrane onto the copper wire, which contained the graphene nanosheets (Figure 2). 


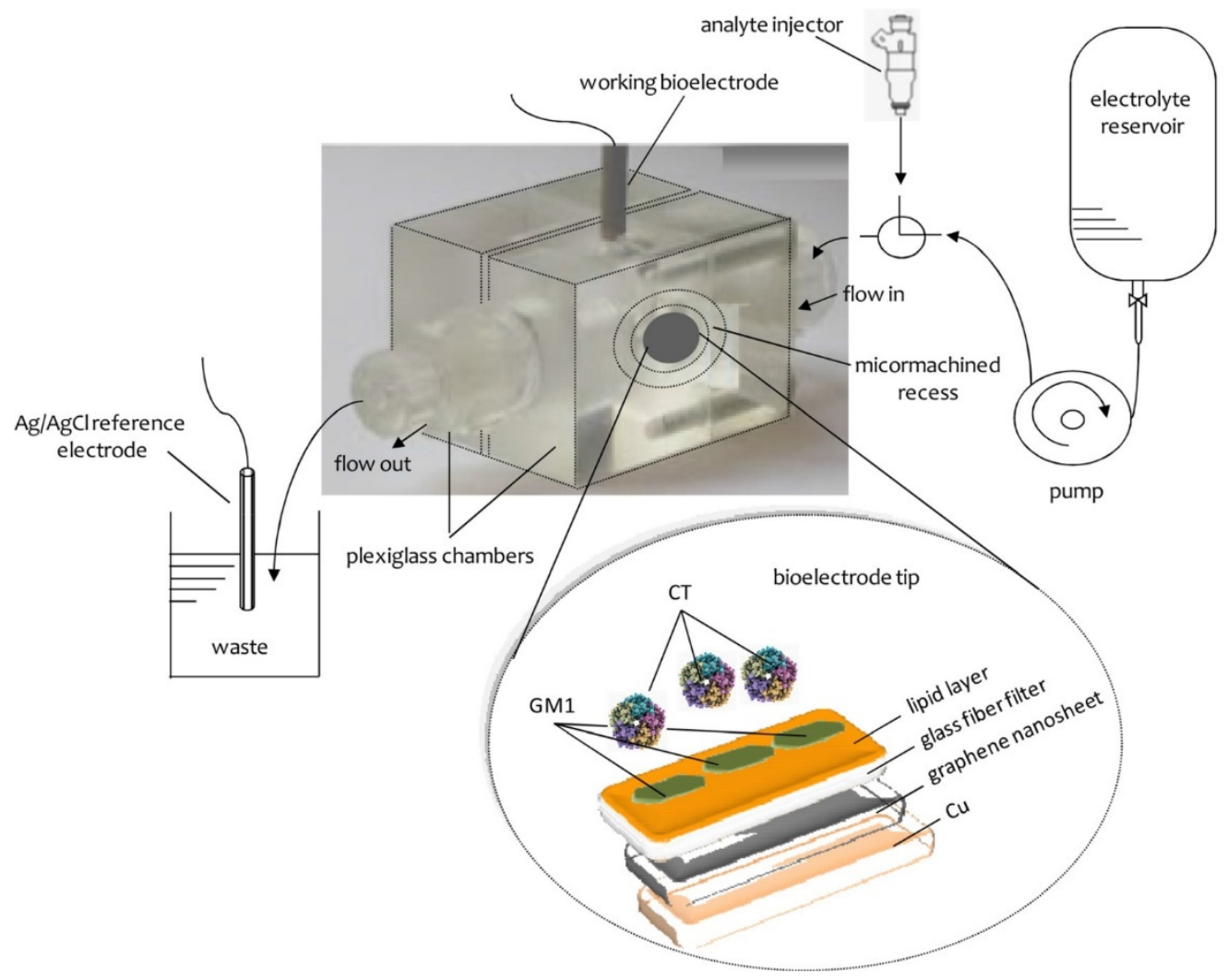

Figure 2. A simplified scheme of the experimental device and the bioelectrode edge surface. Reprinted from Nikoleli et al. [11].

The deposition of tailored phospholipid membranes on graphene using dip-pen nanolithography has also been reported [14]. Dip-pen nanolithography has permitted the multiplexed assembly of phospholipid membranes of different functionalities in close proximity to each other. The membranes are stable in aqueous environments and electronic doping of graphene by charged phospholipids was observed. These results show how phospholipid membranes can be used as a route for non-covalent immobilization on graphene for applications in biosensing and biocatalysis.

Phospholipid membranes of various functionalities were simultaneously self-assembled on arrays of graphene surfaces in a parallel manner using multi-pen lipid dip-pen nano-lithography [15]. The graphene patch assists and restricts the spreading of lipids within itself, removing the need to scan the writing probes. Binding studies established that the lipids retain their functionality.

\section{Potentiometric Applications on Biosensors Based on Lipid Membranes on Graphene Electrodes}

A previous study described a potentiometric urea lipid membrane-based nanosensor incorporated on graphene [3]. The investigation into the structure of graphene nanosheets was completed using atomic force microscopy (AFM) and transmission electron microscopy (TEM) measurements. UV-Vis (Ultraviolet-visible) and Fourier transform infrared (FTIR) spectrophotometry were used to examine the pre- and post-conjugated surfaces of graphene nanosheets. This potentiometric urea nanosensor had good reproducibility, reusability, selectivity, a fast response time of about four seconds, a long shelf life, and high sensitivity with a slope of ca. $70 \mathrm{mV} /$ decade in urea concentrations ranging between $1 \times 10^{-6}$ to $1 \times 10^{-3} \mathrm{M}$.

A cholesterol potentiometric nanosensor was fabricated using immobilization techniques of a stable polymer lipid film that was deposited on graphene [12]. This nanosensor used the enzyme cholesterol oxidase to retain the response toward cholesterol. This device had good reproducibility, 
high selectivity, and excellent sensing capability, with a linear slope curve of ca. $64 \mathrm{mV}$ per decade. Given the biocompatibility between lipid films and human biofluids (e.g., serum and urine), using this device for human blood samples and other biological fluids is possible.

The deposition of tailored phospholipid membranes on graphene using dip-pen nanolithography has been used for the specific binding of streptavidin to biotin-functionalized membranes [14]. The combination of atomic force microscopy and binding experiments yielded a consistent model for the layer organization within phospholipid stacks on graphene.

The interactions of graphene and few-layer graphene (FLG) microsheets with three cell types and with model lipid bilayers by combining coarse-grained molecular dynamics (MD), all-atom MD, analytical modeling, confocal fluorescence imaging, and electron microscopic imaging were investigated [16]. The imaging experiments showed edge-first uptake and complete internalization for a range of FLG samples with a lateral dimension of 0.5 to $10 \mu \mathrm{m}$. In contrast, the simulations showed large energy barriers relative to $k_{B} T$ for membrane penetration by model graphene or FLG microsheets of similar size. More detailed simulations resolved this paradox; the entry has been shown to start at the corners or asperities that are abundant along the irregular edges of the graphene. Local piercing by these sharp protrusions propagates a membrane along the graphene edge avoiding the high energy barrier. This mechanism was suggested to permit the cellular uptake of larger multilayer sheets of $\mu \mathrm{m}$-scale lateral dimension, which is consistent with our multimodal bioimaging results for primary human keratinocytes, human lung epithelial cells, and murine macrophages.

The construction of a potentiometric D-dimer nanosensor on graphene using polymer lipid membranes was reported in the literature [17]. This graphene biosensor was applied to construct a selective and sensitive immunosensor for the determination of D-dimer; this was accomplished by using the mouse anti human D-dimer antibody as the receptor, which was immobilized on stabilized polymer lipid membranes on graphene bioelectrode. The responses toward D-dimer concentrations ranged between $10^{-6}$ to $10^{-3} \mu \mathrm{g} / \mathrm{L}$ and the response times were ca. $15 \mathrm{~s}$. This potentiometric D-dimer device can be constructed easily, with good reproducibility, reusability, selectivity, rapid response times, a long shelf life, and high sensitivity with a linear logarithmic slope of ca. $59 \mathrm{mV} /$ decade in the D-dimer concentration range from $10^{-6}$ to $10^{-3} \mu \mathrm{g} / \mathrm{L}$.

A carbofuran chemosensor based on a graphene electrode using lipid membranes was reported for the determination of carbofuran in real samples of fruits and vegetables [1]. The graphene electrode was used as a basis to develop a chemosensor to sensitively and selectively determine carbofuran using the immobilization technique of an artificial receptor deposited onto stable lipid membranes. The method of preparation of this receptor was previously described [1] and the technique included altering the hydroxyl groups of resorcinarene in phosphoryl groups. The chemosensor responded in the $\mathrm{mM}$ concentration range of carbofuran and had rapid response times of about $20 \mathrm{~s}$. This potentiometric carbofuran chemosensor is constructed easily and is reproducible and sensitive; its response times are very short (20 s), exhibiting a long period of stability with logarithmic concentration response with a slope of $59 \mathrm{mV} /$ decade ranging between $10^{-6}$ and $10^{-3} \mathrm{M}$.

A potentiometric miniature cholera toxin device that was based on a graphene electrode with deposited lipid membranes was reported [18]. Ganglioside GM1, which is the natural receptor for cholera toxin, was mixed with the lipid mixture prior to polymerization. This device had good selectivity and sensitivity with nanomolar detection limits and rapid response times (ca. $5 \mathrm{~min}$ ). The nanosensor was easily constructed and displayed excellent characteristics in terms of reproducibility, reusability, selectivity, and long shelf life. The slope was ca. $60 \mathrm{mV} /$ decade over a logarithmic cholera toxin concentration. The method was applied for the determination of this toxin in water samples of lakes.

A naphthalene acetic acid (NAA) potentiometric device, using the described graphene electrode in which the lipid membrane transducer was deposited, has been described in the literature [2]. The receptor (auxin-binding protein 1) was placed in the lipid mixture prior to polymerization. The selectivity and sensitivity of the response were very good, the detection limits were in the $\mathrm{mM}$ 
range, and the response times were ca. $5 \mathrm{~min}$. The device can be constructed very easily with excellent reproducible results and can be reused many times. The shelf life was very long and the slope of the potentiometric nanosensor was $56 \mathrm{mV}$ /decade of NAA concentration. An evaluation and validation of this device was made by using spiked fruits and vegetables.

A saxitoxin nanosensor based on graphene electrodes with a deposited lipid membrane and in mixture with anti-STX (the natural saxitoxin receptor) was described [19]. Saxitoxin was selectively determined in concentrations ranging between $1 \times 10^{-9}$ and $1 \times 10^{-6} \mathrm{M}$, the response times were between 5 and $20 \mathrm{~min}$, and the detection limit was one nM. This nanosensor was constructed easily with good reproducibility and selectivity. This device can be used many times, is stable at ambient temperatures, and has a long shelf life. The slope of the electrode is $60 \mathrm{mV} /$ decade of saxitoxin. The method was applied in real samples with validation in lake water and shellfish samples. The present technology can be adapted to detecting other toxins and can used to combat bioterrorism.

Supported phospholipid membrane patches were stabilized on graphene surfaces, exhibiting potential for biosensor device construction [20]. Lipid dip-pen nanolithography was applied. Atomic force microscopy and coarse-grained molecular dynamics simulation methods were used to characterize the molecular properties of supported lipid membranes materials such as graphene and graphene oxide [20]. Large differences in the topologies of the film structures were discovered, which depended on the nature of the surface. The addition of water to supported phospholipid films resulted in the reorganization of the membrane structures, with measurable effects on lipid lateral mobility within the films. Reduced lipid ordering within the supported membranes relative to free-standing lipid bilayers were recorded, which were related to the strong hydrophobic interactions between the lipids and graphene. These results provide insight into the molecular effects of graphene and graphene oxide surfaces on lipid films. These investigations will be important when designing these devices for applications in biosensor fields.

\section{Conclusions}

This article reviewed the construction of biosensors based on polymer lipid membranes on graphene electrodes and their potentiometric applications. These applications include the rapid detection of a wide range of toxic substances in foods, environmental pollutants, and compounds of biomedical importance. These biosensors have shown adequate selectivity, sensitivity, reproducibility, rapid response times, while being easy to construct. The person who uses these devices can be non-skilled and these nanosensors can be used for in-field measurements. Research is now being directed to the use of nanotechnological advances to construct portable nanosensors with even better characteristics that can be used for the rapid determination of analytes, such as allergens, antibiotics, hydrazines, polycyclic aromatic hydrocarbons, polycyclic carbamates, and their residues. These investigations will introduce nanodevices that can be used for the field and in situ determination of toxicants in foods and the environment, which will in turn improve human safety.

Conflicts of Interest: The authors declare no conflict of interest.

\section{References}

1. Bratakou, S.; Nikoleli, G.-P.; Nikolelis, D.P.; Psaroudakis, N. Development of a potentiometric chemical sensor for the rapid detection of carbofuran based on air stable lipid films with incorporated calix[4]arene phosphoryl receptor using graphene electrodes. Electroanalysis 2015, 27, 2608-2613. [CrossRef]

2. Bratakou, S.; Nikoleli, G.-P.; Siontorou, C.G.; Nikolelis, D.P.; Tzamtzis, N. Electrochemical biosensor for naphthalene acetic acid in fruits and vegetables based on lipid films with incorporated auxin-binding protein receptor using graphene electrodes. Electroanalysis 2016, 28, 2171-2177. [CrossRef]

3. Nikoleli, G.-P.; Israr, M.Q.; Tzamtzis, N.; Nikolelis, D.P.; Willander, M.; Psaroudakis, N. Structural characterization of graphene nanosheets for miniaturization of potentiometric urea lipid film based biosensors. Electroanalysis 2012, 24, 1285-1295. [CrossRef] 
4. Nikolelis, D.P.; Siontorou, C.G.; Andreou, V.G.; Viras, K.G.; Krull, U.J. Bilayer lipid membranes as electrochemical detectors for flow injection immunoanalysis. Electroanalysis 1995, 7, 1082-1089. [CrossRef]

5. Nikolelis, D.P.; Mitrokotsa, M. Stabilized lipid film based biosensor for atenolol. Biosens. Bioelectron. 2002, 17, 565-572. [CrossRef]

6. Nikolelis, D.P.; Theoharis, G. Biosensor for dopamine based on stabilized lipid films with incorporated resorcin[4]arene receptor. Bioelectrochemistry 2003, 59, 107-112. [CrossRef]

7. Nikolelis, D.P.; Drivelos, D.A.; Simantiraki, M.G.; Koinis, S. An Optical Spot Test for the Detection of Dopamine Human Urine Using Stabilized in Air Lipid Films. Anal. Chem. 2004, 76, 2174-2180. [CrossRef] [PubMed]

8. Nikolelis, D.P.; Mitrokotsa, M. Rapid electrochemical detection of propranolol and metoprolol in pharmaceutical preparations using stabilized lipid films. Electroanalysis 2004, 16, 741-747. [CrossRef]

9. Nikolelis, D.P.; Raftopoulou, G.; Chatzigeorgiou, P.; Nikoleli, G.-P.; Viras, K. Optical portable biosensors based on stabilized lipid membrane for the rapid detection of doping materials in human urine. Sens. Actuators B Chem. 2008, 130, 577-582. [CrossRef]

10. Nikoleli, G.-P.; Nikolelis, D.P.C. Methenitis Construction of a simple optical sensor based on air stable lipid film with incorporated urease for the rapid detection of urea in milk. Anal. Chim. Acta 2010, 675, 58-63. [CrossRef] [PubMed]

11. Nikoleli, G.-P.; Siontorou, G.C.; Nikolelis, D.P.; Karapetis, S. Lipid Membrane Nanosensors for Environmental Monitoring: The Art, the Opportunities, and the Challenges. Sensors 2018, 18, 284. [CrossRef] [PubMed]

12. Nikoleli, G.-P.; Ibupoto, Z.H.; Nikolelis, D.P.; Likodimos, V.; Psaroudakis, N.; Tzamtzis, N.; Willander, M.; Hianik, T. Potentiometric cholesterol biosensing application of graphene electrode with stabilized polymeric lipid membrane. Cent. Eur. J. Chem. 2013, 11, 1554-1561. [CrossRef]

13. Hernandez, Y.; Nicolosi, V.; Lotya, M.; Blighe, F.M.; Sun, Z.; De, S.; McGovern, I.T.; Holland, B.; Byrne, M.; Gun'ko, Y.K.; et al. High-yield production of graphene by liquid-phase exfoliation of graphite. Nat. Nanotechnol. 2008, 3, 563-568. [CrossRef] [PubMed]

14. Hirtz, M.; Oikonomou, A.; Georgiou, T.; Fucks, H.; Vijayaghavan, A. Multiplexed biomimetic lipid membranes on graphene by dip-pen nanolithography. Nat. Commun. 2013, 4, 2591. [CrossRef] [PubMed]

15. Hirtz, M.; Oikonomou, A.; Clark, N.; Kim, Y.-J.; Fucks, H.; Vijayaghavan, A. Self-limiting multiplexed assembly of lipid membranes on large-area graphene sensor arrays. Nanoscale 2016, 8, 15147-15151. [CrossRef] [PubMed]

16. Li, Y.; Yuan, H.; Hurt, R.H.; Kane, A.B.; Gao, H. Graphene microsheets enter cells through spontaneous membrane penetration at edge asperities and corner sites. Proc. Natl. Acad. Sci. USA 2013, 110, 12295-13000. [CrossRef] [PubMed]

17. Nikoleli, G.-P.; Psaroudakis, N.; Nikolelis, D.P.; Tzamtzis, N. A Selective Immunosensor for D-dimer Based on Antibody Immobilized on a Graphene Electrode with Incorporated Lipid Films. Electroanalysis 2014, 26, 1522-1527. [CrossRef]

18. Karapetis, S.; Nikoleli, G.-P.; Siontorou, C.G.; Nikolelis, D.P.; Tzamtzis, N.; Psaroudakis, N. Development of an electrochemical biosensor for the rapid detection of cholera toxin based on air stable lipid films with incorporated ganglioside GM1 using graphene electrodes. Electroanalysis 2016, 28, 1584-1590. [CrossRef]

19. Bratakou, S.; Nikoleli, G.-P.; Siontorou, G.C.; Nikolelis, D.P.; Karapetis, S.; Tzamtzis, N. Development of an electrochemical biosensor for the rapid detection of saxitoxin based on air stable lipid films with incorporated Anti-STX using graphene electrodes. Electroanalysis 2017, 29, 990-997. [CrossRef]

20. Willems, N.; Urtizberea, A.; Verre, A.F.; Iliut, M.; Lelimousin, M.; Hirtz, M.; Vijayaraghavan, A.; Sansom, M.S.P. Biomimetic phospholipid membrane organization on graphene and graphene oxide suefaces: A molecular dynamics simulation study. ACS Nano 2017, 11, 1613-1625. [CrossRef] [PubMed]

(C) 2018 by the authors. Licensee MDPI, Basel, Switzerland. This article is an open access article distributed under the terms and conditions of the Creative Commons Attribution (CC BY) license (http://creativecommons.org/licenses/by/4.0/). 